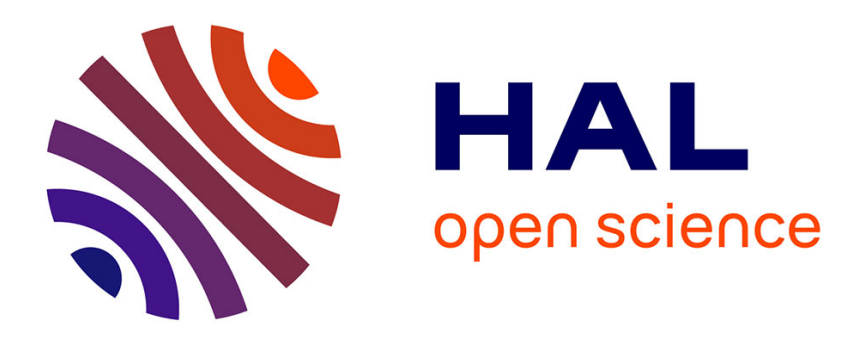

\title{
A rheological description of amylose gelation
}

\author{
J.L. Doublier, L. Choplin
}

\section{To cite this version:}

J.L. Doublier, L. Choplin. A rheological description of amylose gelation. Carbohydrate Research, 1989, 193, pp.215-226. hal-02722452

\section{HAL Id: hal-02722452 \\ https://hal.inrae.fr/hal-02722452}

Submitted on 1 Jun 2020

HAL is a multi-disciplinary open access archive for the deposit and dissemination of scientific research documents, whether they are published or not. The documents may come from teaching and research institutions in France or abroad, or from public or private research centers.
L'archive ouverte pluridisciplinaire HAL, est destinée au dépôt et à la diffusion de documents scientifiques de niveau recherche, publiés ou non, émanant des établissements d'enseignement et de recherche français ou étrangers, des laboratoires publics ou privés. 


\section{A RHEOLOGICAL DESCRIPTION OF AMYLOSE GELATION}

JEAN-LOUIS DOUblier*+ AND LIONEL ChOPLIN

Department of Chemical Engineering and CERSIM (Centre de Recherches en Sciences et Ingénierie des Macromolécules), Université Laval; Québec, QC, GIK $7 P 4$ (Canada)

(Received August 23rd, 1988; accepted for publication, April 22nd, 1989)

\section{ABSTRACT}

Amylose gels of low concentration (1-3\%) were prepared by neutralization of alkaline solutions of amylose. The kinetics of amylose gelation were studied by small-amplitude oscillatory shear experiments. The evolution of the storage modulus $\left(G^{\prime}\right)$ and the loss modulus $\left(G^{\prime \prime}\right)$ involved first an induction period (5-600 min, depending on the amylose concentration and ionic strength) where the medium remained clear and no variation of $G^{\prime}(t)$ and $G^{\prime \prime}(t)$. was evident. Then there was a sharp increase in $G^{\prime}$ and a slight increase in $G^{\prime \prime}(t)$. The point where the $G^{\prime}(t)$ and $G^{\prime \prime}(t)$ curves separated was the gel time (g.t.) and was related to the time when the medium became cloudy (c.t., cloud time). The second step lasted 10-100 min, depending upon the solution conditions. The third period was characterized by a slow evolution of $G^{\prime}(t)$ and no equilibrium value was reached after 15 h. G.t., c.t., and the final value of $G^{\prime}$ (for 15-h gels) were strongly dependent on the concentration of amylose and the content of $\mathrm{KCl}$. The limiting concentration $\left(C_{0}\right)$ of amylose for gelation was $0.9 \%$, corresponding to $C_{0}[\eta]=0.64$. The effect of ionic strength on the kinetics of amylose gelation and gel stiffness could be interpreted II on the basis of a phase separation process.

(a)

\section{INTRODUCTION}

The amylose component of starch is a linear $(1 \rightarrow 4)$ - $\alpha$-D-glucan, whereas amylopectin is highly branched with $\alpha-(1 \rightarrow 4)$ and $\alpha-(1 \rightarrow 6)$ linkages. Amylose accounts for only $20-32 \%$ of normal starch, depending upon the botanical origin, but it makes a major contribution to the properties. Heating an aqueous dispersion of starch above $60-70^{\circ}$ yieids a suspension with swollen particles, composed of amylopectin, in a continuous phase composed of amylose. On cooling, the starch paste yields a composite gel the continuous matrix of which arises primarily from amylose gelation ${ }^{1,2}$. This process is also referred to as amylose retrogradation and

*Author for correspondence.

TPermanent address: Institut National de la Recherche Agronomique, B.P. 527, F-44026 Nantes 03, France. 
is of primary importance in the determination of textural properties of starchy food products.

Until recently, little was known about the gelation process of amylose During the formation of starch gels, a B-type crystalline pattern was reported to develop ${ }^{3.4}$. On the basis of turbidity measurements, rheology, dilatometry, and $X$ ray measurements, on $2-7 \%$ amylose gels, it was suggested ${ }^{5.6}$ that gelation involves a phase separation into polymer-rich and polymer-deficient regions. Development of the gel network [as estimated by the increase in the shear modulus $\left(G^{\prime}\right)$ ] and phase separation are related. Because these phenomena occur in the early stages of the gelation process, they do not seem to be related to crystallinity which develops much more slowly. However, the kinetics of phase separation and gel formation are difficult to describe accurately for these concentrated systems. It was suggested ${ }^{5,6}$ that such a process can occur provided the concentration $\left(C^{*}\right)$ for coil overlap is reached, and it was inferred that $C_{0}$, the minimum concentration required for gelation, should be of the order of $C^{*}$. This point was contradicted recently in a study with amyloses of different molecular weights ${ }^{7-9}$ where it was reported that gelation can occur for concentrations well below $C^{*}$, this parameter being determined from viscosity measurements ${ }^{9}$. Surprisingly, $C_{0}$ varied only slightly $(0.8-$ $1.1 \%$ ) with the molecular weight provided the d.p. was $>300^{8,9}$. No systematic correspondence was seen between the formation of a gel, as assessed from the variation in $G^{\prime}$, and the development of turbiditys. It was suggested" that "amylose aggregation is the adoption of double-helix structures, followed by helix-helix aggregation, thereby leading to turbidity effects and, for sufficiently long amylose chains, cross-linked networks and gelation".

Other studies of amylose retrogradation from neutral aqueous $<0.3 \%$ solution involved light-scattering experiments ${ }^{10-12}$. A slow increase in turbidity was reported, showing that stability is never achieved under neutral conditions so that retrogradation can occur in very dilute conditions. Such instability arises from macromolecular aggregation which is probably the same process as that yielding gelled amylose. More recently, microscopy ${ }^{13}$ on $0.4 \%$ amylose in aqueous $\mathrm{KCl}$ revealed that particles of uniform size arose from amylose retrogradation; these particles displayed a weak B-type crystalline structure.

There is a gap between observations for highly dilute solutions $(<0.3 \%)$ where slow changes in turbidity were displayed but without gelation, and for concentrated media $(>2 \%)$ where the gelation process is too rapid to be described accurately. The boundary between gel formation and precipitation lies in this gap. It is also in the vicinity of $C_{0}$, the critical concentration for gelation to occur, that the sol-gel transition takes place sufficiently slowly for accurate measurement. The present investigation was undertaken to describe the formation of amylose gels of low concentration (1-2\%). 
Materials. - The amylose sample was AVEBE amylose-V from potato starch. Samples of the same origin were used by Kitamura et al. ${ }^{13}$ and by Hayashi et al. ${ }^{14.15}$ in other studies of amylose gelation. The intrinsic viscosity in $\mathrm{KCl}(0.33 \mathrm{M})$ at $25^{\circ}$ was $70 \mathrm{~mL} / \mathrm{g}$, yielding a viscosity-average molecular weight $\left(\bar{M}_{\mathrm{v}}\right)$ of 390,000 according to the equation proposed for amylose in pure aqueous solution ${ }^{16}$ or in the presence of $\mathrm{KCl}^{17.18}$.

Preparation of gels. - Gels were prepared under isothermal conditions by neutralization of alkaline solutions ${ }^{13}$. This method was preferred to the regeneration $^{5.6}$ of an amylose solution from dispersions of an amylose/butanol complex in hot water or by heating aqueous amylose dispersions ${ }^{7-9}$ up to $150-160^{\circ}$. The main reason was that, under these conditions, the temperature history is a critical parameter for the sol-gel transition and the final characteristics of gels $5,7.8$.

Stock $7.5 \%(\mathrm{w} / \mathrm{w})$ solutions of amylose in $\mathrm{M} \mathrm{KOH}$ were stored at $4^{\circ}$. No macromolecular degradation had occurred after several weeks. Gels were prepared by adding $\mathrm{M}$ or $0.3 \mathrm{M} \mathrm{HCl}$ dropwsie to the solution of amylose. The final $\mathrm{pH}$ was 6.5-7.5.

Rheology. - Small-amplitude oscillatory-shear experiments were performed at $25^{\circ}$ with a Rheometrics System-4 Mechanical rheometer using the cone and plate geometry (diameter, $5 \mathrm{~cm}$; angle, $1^{\circ}$ ). Measurements were carried out at $5 \mathrm{rad} / \mathrm{s}$ and strain amplitudes of 5 or $10 \%$ that were well below the limit of the linear viscoelasticity domain for final 15 -h gels. Characteristics of the final gels were also determined through measurements of $G^{\prime}$ and $G^{\prime \prime}$ as a function of frequency between $10^{-2}$ and $10^{+2} \mathrm{rad} / \mathrm{s}$.

Measurements of turbidity. - Development of turbidity was monitored by measuring absorbance variations as a function of time at $640 \mathrm{~nm}$, using a u.v./visible spectrophotometer at $25^{\circ}$ unless noted otherwise.

\section{RESULTS}

A typical example of the evolution of the storage modulus $\left(G^{\prime}\right)$ and the loss modulus $\left(G^{\prime \prime}\right)$ together with the variation in turbidity during the gelation proces is shown in Fig. 1 for $1.78 \%$ amylose in $0.5 \mathrm{M} \mathrm{KCl}$. The solution was optically clear until after neutralization and became cloudy after a lag time of $40 \mathrm{~min}$. Beyond this point, turbidity increased and the medium was totally opaque after $\sim 2 \mathrm{~h}$. On the other hand, a sharp increase in $G^{\prime}$ was seen beyond $\sim 80 \mathrm{~min}$. The first $90 \mathrm{~min}$ of the process are plotted in expanded form in Fig. 2, which shows that the evolution of $G^{\prime}$ takes place earlier than suggested in Fig. 1. During the first $35 \mathrm{~min}, G^{\prime}$ and $G^{\prime \prime}$ were of the order of $0.05 \mathrm{~Pa}$ and at the limit of sensitivity of the measuring system. After $35 \mathrm{~min}, G^{\prime}$ increased steadily to $70 \mathrm{~min}$ and then sharply, but $G^{\prime \prime}$ increased much more slowly. The early increase in $G^{\prime}(t)$ paralleled the increase in turbidity. The variation of $G^{\prime}$ covered more than three orders of magnitude, 


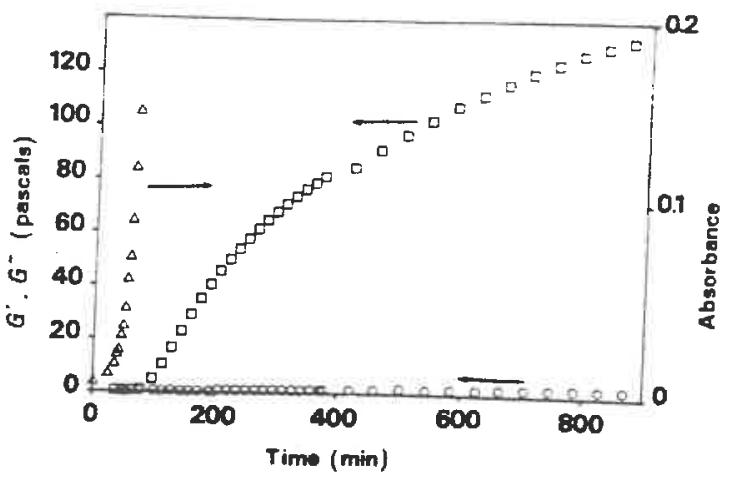
Fig. 1. Variation of $G^{\prime}(\square)$ and $G^{\prime \prime}(O)$ as a function of time at $25^{\circ}$ for $1.78 \%$ amylose in $0.5 \mathrm{M} \mathrm{KCl}$.
Comparison with the evolution of turbidity $(\triangle$, absorbance at $640 \mathrm{~nm})$.

whereas that of $G^{\prime \prime}$ was approximately one order. The relative evolution of both moduli presented semi-logarithmically in Fig. 3 shows the dramatic evolution of $G^{\prime}$ during the first $200 \mathrm{~min}$ and then a slight and continuous increase. Although a steady state value was not obtained after $15 \mathrm{~h}$, this time was chosen for the final value of $G^{\prime}$ for practical reasons. The point of separation of the curves for $G^{\prime}$ and $G^{\prime \prime}$ in Fig. 2 is defined as the gel time (g.t.) based on the chemical gelation of synthetic polymers ${ }^{19}$ and it is generally accepted to occur somewhere in the vicinity of the $G^{\prime}-G^{\prime \prime}$ crossover $^{20}$. This was also demonstrated to be a useful definition for the physical gelation of biopolymers ${ }^{21}$. Similarly, the cloud time (c.t.) is defined arbitrarily as the point of departure from the baseline of the turbidity curve (see Fig. 2) and is the time when the particles become large enough to scatter incident light. This parameter was taken as a measure of the rate of the aggregation process. The fact that, in Fig. 2, the c.t. was close to the g.t. confirms that the formation of the network and the development of the turbidity take place within the same time-
scale ${ }^{5,6}$.

Figs. 4 and 5 show variations in $G^{\prime}$ plotted on semi-log scales for $1-2.75 \%$

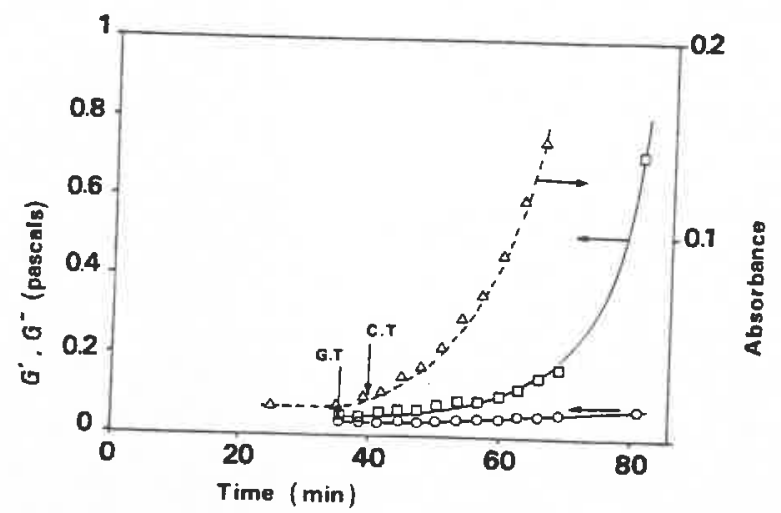

Fig. 2. As in Fig. 1 for the first $90 \mathrm{~min}$. Arrows show gel time and cloud time (see text). 


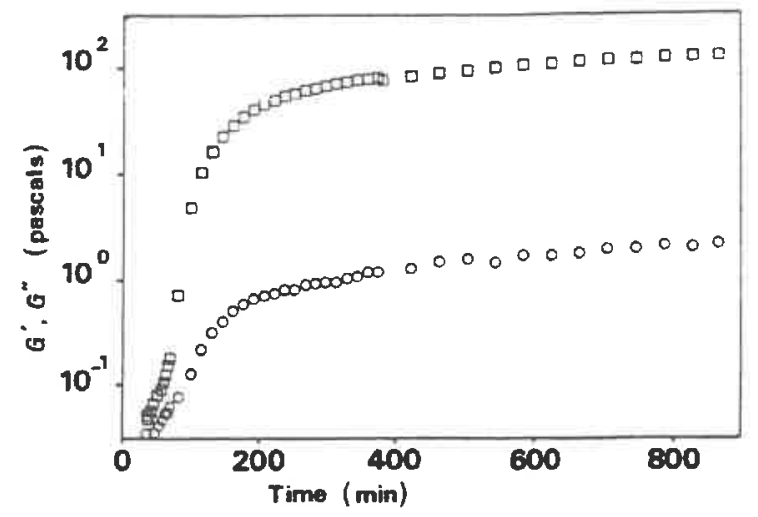

Fig. 3. $G^{\prime}(t)$ and $G^{\prime \prime}(t)$ on a semi-log scale. Symbols as in Figs. 1 and 2.

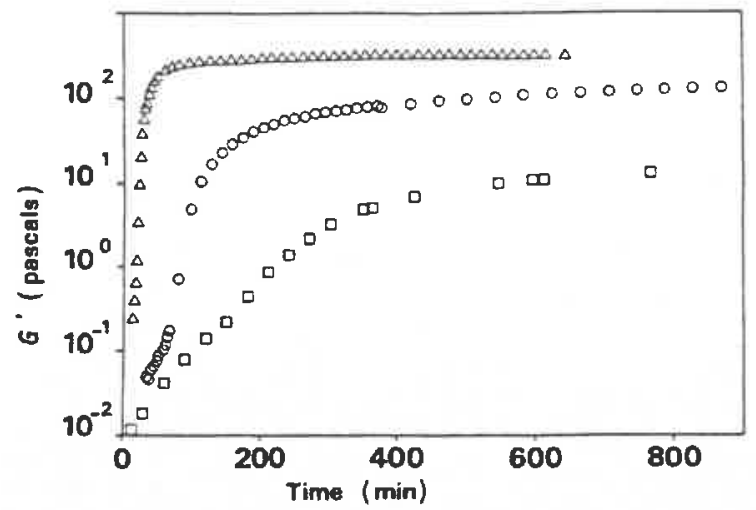

Fig. 4. Variation of $G^{\prime}$ as a function of time for amylose in $0.5 \mathrm{M} \mathrm{KCl}$ at $25^{\circ}: \square, 1.37 \% ; 0,1.78 \% ; \Delta$, $2.75 \%$.

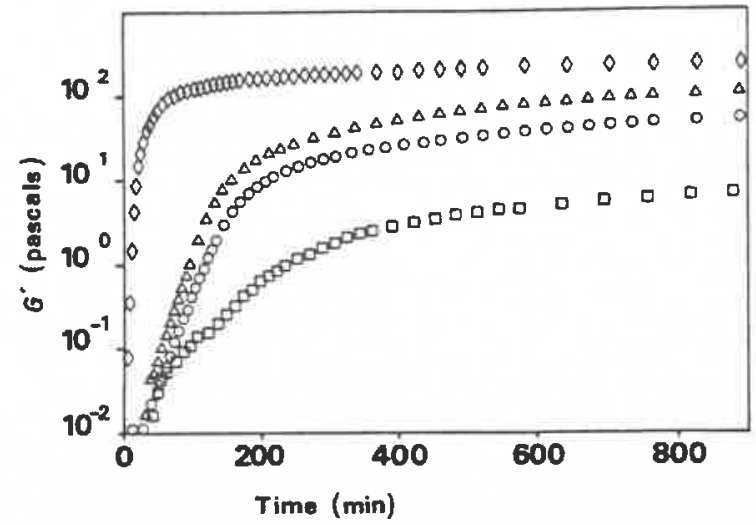

Fig. 5. Variation of $G^{\prime}$ as a function of time for amylose in $0.2 \mathrm{M} \mathrm{KCl}$ at $25^{\circ}: \square, 1.03 \% ; 0,1.33 \% ; \Delta$, $1.48 \% ; \diamond, 1.78 \%$. 


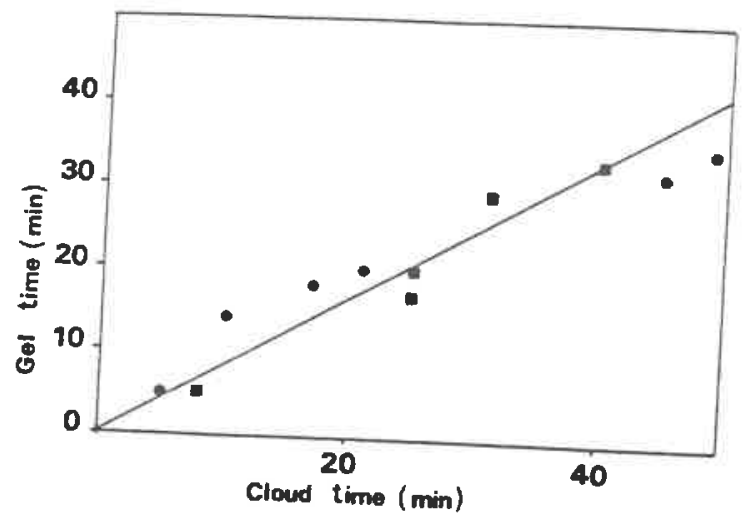

Fig. 6. Gel time (g.t.) as a function of cloud time (c.t.) in $\mathrm{KCl}: \mathrm{m}, 0.5 \mathrm{M} \mathrm{KCl} ; 0,0.2 \mathrm{M}$

amylose in 0.5 and $0.2 \mathrm{M} \mathrm{KCl}$. For the sake of clarity, the variations in $G^{\prime \prime}$ are not shown, but they show the same trend as in Fig. 3. For the highest concentrations of slightly beyond the g.t., reflecting first step and then its value dropped to zero below the resolution of the instring the fact that the loss angle $\delta\left(\operatorname{tg} \delta=G^{\prime \prime} / G^{\prime}\right)$ was but remained $\sim 100$ times lower thent. Beyond $12 \mathrm{~h}, G^{\prime \prime}$ could be measured again times higher in 0.2 than in $0.5 \mathrm{M}$ than $G^{\prime}$. At equivalent concentration, $G^{\prime}$ was 5 much stiffer. of the amylose and the ionic strengeen c.t. and g.t. whatever the concentration correlation line $(0.8)$ indicates the below). A marked dependence on g.t. to be slightly shorter than the c.t. (see the ultimate value of $G^{\prime}$ as illustrated in estimated from the c.t., varies as a function 7 where the induction period, as respectively.

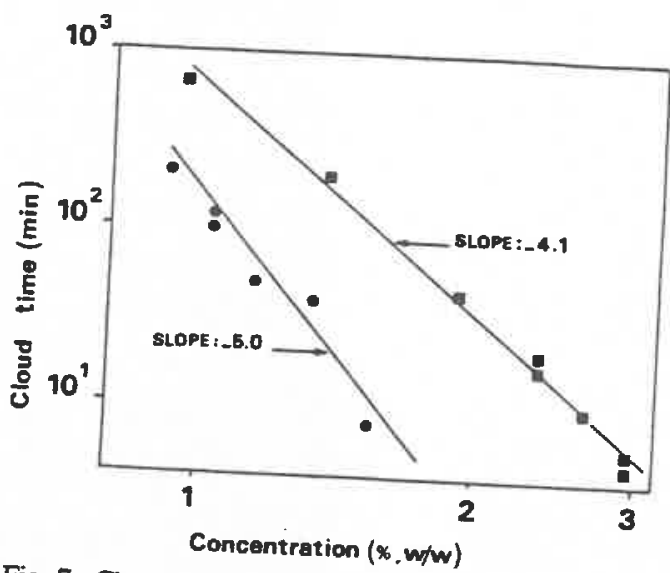

Fig. 7. Cloud time (c.t.) as a function of concentration in $\mathrm{KCl}: \mathrm{a}, 0.5 \mathrm{M} \mathrm{KCl} ; \ominus, 0.2 \mathrm{M}$. 


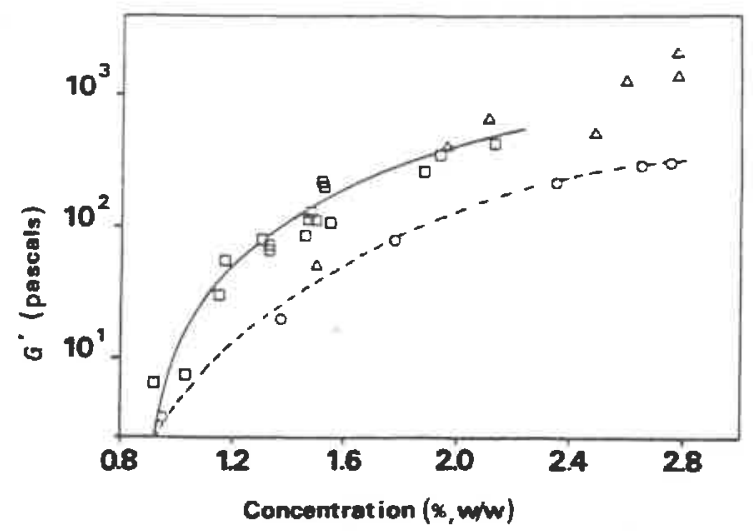

Fig. 8. $G^{\prime}$ as a function of concentration for 15 -h old gels in $\mathrm{KCl}: 0,0.5 \mathrm{M} ; \square, 0.2 \mathrm{M} ; \Delta$, data of Ellis and Ring ${ }^{6}$.

Variations of the ultimate value of $G^{\prime}$ as a function of concentration are shown in Fig. 8. Values reported ${ }^{6}$ for concentrations $<3 \%$ are also plotted, but the sample of amylose was of different origin, had a higher molecular weight, and the gels were prepared by the dispersion of an amylose/butanol complex in hot water. The latter data are close to the present results at an ionic strength of $0.2 \mathrm{M}$ but show a larger scatter. The dependence of the final values of $G^{\prime}$ on concentration was marked, particularly at the lowest concentrations. The notable difference in stiffness between the gels in 0.2 and $0.5 \mathrm{M} \mathrm{KCl}$ is also seen clearly. The value of $G^{\prime}$ extrapolates to zero for a concentration of $0.9 \%$. This value $\left(C_{0}\right)$ was the same for both ionic strengths examined and can be taken as an estimate of the lowest concentration where gelation is possible.

Fig. 9 shows the frequency dependence of the final values of $G^{\prime}$ and $G^{\prime \prime}$ for two of the lowest concentrations. More-concentrated gels yielded a similar pattern

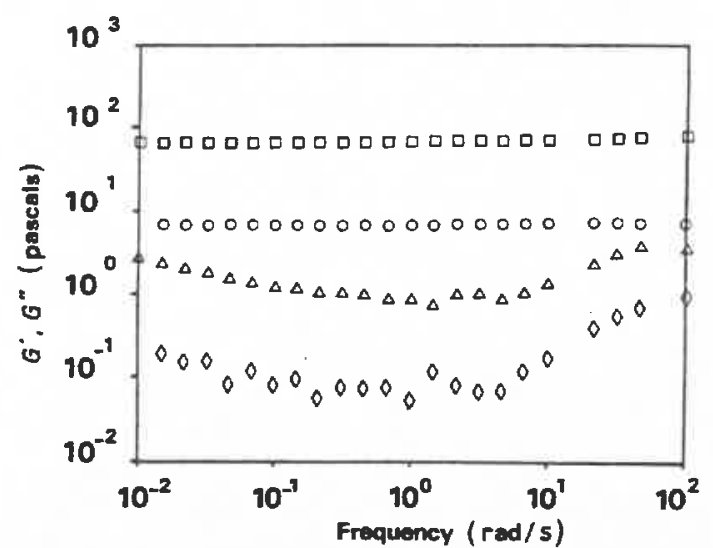

Fig. 9. $G^{\prime}$ and $G^{\prime \prime}$ as a function of frequency: $1.03 \%$ amylose, $O, G^{\prime} ; \diamond, G^{\prime \prime} ; 1.33 \%$ amylose, $\square, G^{\prime} ; \triangle$, $G^{\prime \prime} ; 0.2 \mathrm{M} \mathrm{KCl}$. 


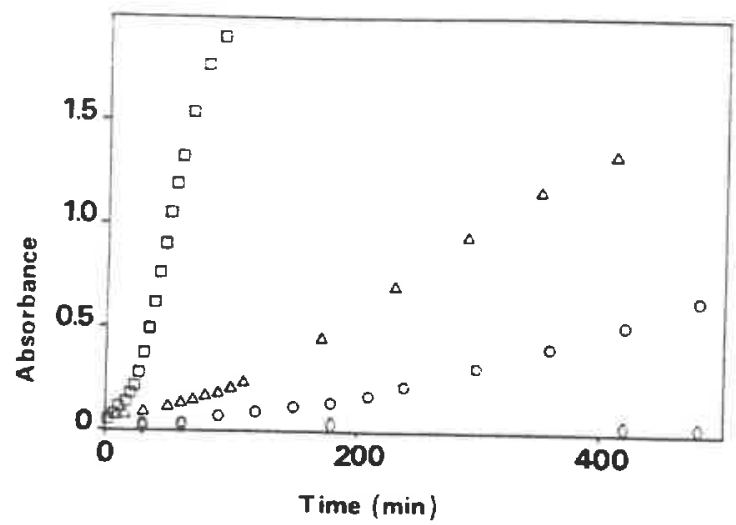

Fig. 10. Variation in turbidity as a function of time for $1.6 \%$ amylose in $0.2 \mathrm{M} \mathrm{KCl}: \square, 25^{\circ} ; \Delta, 35^{\circ} ; 0$, $40^{\circ} ; \diamond, 42^{\circ}$.

with much higher values of $G^{\prime}$ and $G^{\prime \prime}$. There was no variation in $G^{\prime}$ within the experimental range of frequency, that is between $10^{-2}$ and $10^{+2} \mathrm{rad} / \mathrm{s}$, and the value of $G^{\prime}$ was 1 or 2 orders of magnitude greater than that of $G^{\prime \prime}$. These two features are used classically as criteria for the definition of "true" gels. In contrast, with a "pseudo" gel, $G^{\prime}$ would vary slightly with frequency and the $G^{\prime \prime} / G^{\prime}$ ratio would be of the order of 0.1 or more ${ }^{22}$. The value of $G^{\prime \prime}$ was a minimum at a frequency of $\sim 1$ $\mathrm{rad} / \mathrm{s}$ as has been observed for other biopolymer gels (i.e., gelatin ${ }^{23}$ ).

Fig. 10 illustrates the effect of temperature on the development of turbidity for a $1.6 \%$ amylose gel in $0.2 \mathrm{M} \mathrm{KCl}$. When temperature increased from $25^{\circ}$ to $42^{\circ}$, the development of cloudiness was slowed dramatically, the c.t. being almost infinite at $42^{\circ}$; this was defined as the cloud limiting temperature (c.l.t.) and the variations for concentrations ranging from 1 to $1.8 \%$ were determined. There was a slight concentration dependence; the c.l.t. varied from $35^{\circ}$ to $45^{\circ}$ when the concentration increased from $1 \%$ to $1.8 \%$. These values are slightly higher than those reported ${ }^{15}$ in water/methyl sulfoxide $(90: 10)$ systems ( $35^{\circ}$ whatever the concentration between 1 and $1.5 \%$ ).

\section{DISCUSSION}

The present data show that the gelation of amylose can be investigated easily at low concentration conditions just above $C_{0}$. Even for the more dilute conditions $(\sim 1 \%)$, the amylose gels were smooth and did not display any syneresis even after storage at $25^{\circ}$ for several days. Moreover, the frequency dependence of $G^{\prime}$ as well as the $G^{\prime \prime} / G^{\prime}$ ratio, whatever the concentration, were consistent with the definition ${ }^{22}$ of "true" gels.

Highly expanded amylose coils exist in alkaline media ( $\mathrm{pH} 13-14)$, these being good solvents for amylose $\mathrm{e}^{24}$. However, unperturbed dimensions are adopted at $\mathrm{pH} 7$ and $25^{\circ}$, whatever the ionic strength ${ }^{16-18}$, as displayed by the exponent (a 
$=0.5$ ) of the Mark-Houwink relationship. On decreasing the $\mathrm{pH}$ from 14 to 7 , amylose molecules change from a highly expanded coil to the compact one. This change probably occurs at $\mathrm{pH} 11-12$ as shown by viscometric measurements ${ }^{17,25}$. Thus, a neutral medium is a poor solvent for amylose and aggregation at finite concentration can occur. The kinetics of the aggregation process in highly dilute solutions $(<0.3 \%)$ have been investigated by light-scattering experiments ${ }^{12.26}$ and aggregation was reported to proceed as soon as the alkaline solution was neutralized $^{26}$. The size of aggregates was linearly dependent on time. It is likely that aggregation at higher concentration $(>1 \%)$ occurs by the same mechanisms. The study of the development of turbidity (Fig. 10) as a function of time at different temperatures allows a good estimation of the c.l.t., which increased from $35^{\circ}$ to $45^{\circ}$ when concentration increased from 1 to $1.8 \%$. The c.l.t.-concentration dependence can be used to describe the co-existence line in the phase diagram of the amylose-water system. The gelation experiments reported here for concentrations between 1 and $3 \%$ were performed at $25^{\circ}$, i.e., below this co-existence line. Thus, changing the $\mathrm{pH}$ from 14 to 7 brings the system into the two-phase region of the phase diagram, the polymer solubility is reduced, and demixing occurs. Such a phase separation or aggregation of polymer molecules may result from liquid-liquid phase transitions and is usually observed for the physical gelation of synthetic polymers ${ }^{27}$. Studies of polystyrene, for example, have indicated that gelation occurred, provided that the solution was brought below the $\theta$-temperature within the miscibility gap ${ }^{28,29}$. Consequently, gels were obtained through crystallization which followed phase separation ${ }^{29}$.

The data in Fig. 1 are in qualitative agreement with those reported ${ }^{5,27}$ for $2.4 \%$ amylose gels. Fig. 1 is consistent with Fig. 23 in ref. 27 for the evolution of turbidity and $G^{\prime}$. Despite the fact that the gels used here were prepared in a different way, the same overall process was observed, i.e., a significant increase of $G^{\prime}$ when turbidity was fully developed. The good sensitivity of the rheometer allowed, as illustrated in Fig. 2, confirmation of the suggestion ${ }^{5,6}$ that $G^{\prime}$ increases within the same time-scale as the appearance of cloudiness. This finding supports the hypothesis that gel formation was the result of a phase separation process.

The process of amylose gelation at $25^{\circ}$ can be separated into three steps. First, an induction period when the medium remains clear, $G^{\prime}$ and $G^{\prime \prime}$ being actually non-measurable $(<0.05 \mathrm{~Pa})$. Aggregation of polymer molecules proceeds as soon as neutral $\mathrm{pH}$ is reached, but the size of aggregates is too small for the solution to become cloudy and to be detected by the spectrophotometer. Thus, it is at the beginning of this period that phase separation takes place, giving rise to polymerrich and polymer-poor phases ${ }^{5,6}$. When sufficiently large particles are formed, turbidity develops. The second step then begins at this time when $G^{\prime}>G^{\prime \prime}$. The correspondence between the c.t. and the g.t., as seen in Fig. 2, was found whatever the concentration and the ionic strength, as illustrated in Fig. 6 . The slope of the straight line is lower than $1(0.8)$, showing that the g.t. is reached slightly before cloudiness appears. This does not mean that gelation takes place before phase 
separation since, as discussed above, as soon as the solution is neutralized, conditions are fulfilled for phase separation to occur and physical gelation can take place as with synthetic polymers ${ }^{27-29}$. This correlation clearly indicates that both phenomena are related. The initial increase of $G^{\prime}$ (from 0.05 to $0.2 \mathrm{~Pa}$ ) in the second period lasts $\sim 35 \mathrm{~min}$ in the example of Fig. 2 (from 35 to $70 \mathrm{~min}$ ). This slow increase of $G^{\prime}$ suggests that the polymer-rich phase has become continuous, giving rise to a tenuous network. Such a process can arise when the nucleation density is high or when spinodal phase separation occurs ${ }^{27}$. This tenuous network is then consolidated by a further aggregation of amylose chains within the polymer-rich phase. This aggregation is probably combined with a slow crystallization that results in the sharp increase in $G^{\prime}$ after the initial step (beyond $70 \mathrm{~min}$ for the example in Fig. 2). Such a crystallization process could not be observed in our gels from classical X-ray diffraction patterns owing to the high dilution of amylose. However, Kitamura $e t$ al. ${ }^{10}$ reported a weak B-type pattern for concentrations as low as $0.4 \%$ in 8 -day old amylose gels in $\mathrm{KCl}$. The end of the second period can be roughly estimated at the inflection point of the $G^{\prime}(t)$ curve in linear co-ordinates. The values of $G^{\prime}$ at this stage range from 1 to $10 \mathrm{~Pa}$, depending upon the concentration of the amylose. The duration of this second phase also depends on concentration and varies from $\sim 10$ to $100 \mathrm{~min}$ when concentration increases from 1 to $2 \%$ in $0.2 \mathrm{M} \mathrm{KCl}$. It is at the end of the second period that the gel is completely opaque. The third period is characterized by a slowing of the rate of increase in $G^{\prime}$. However, a steady value is not reached even after $15 \mathrm{~h}$. This period is probably governed by a further crystallization of the amylose chains, but diffusion of macromolecules is slowed due to the
high viscosity of the medium.

The overall variation of $G^{\prime}$ is typical of the pattern expected from theories of gelation $^{31}$. The induction period corresponds to an increase of the size of the particles that aggregated to form a network within which aggregation continues. The present data allow a refinement of previous results $5,6,30$ and are consistent with a quite general phenomenon for synthetic polymers where the network structure depends on the phase separation process provided the system is brought into the two-phase region of the phase diagram ${ }^{27-29}$. This conclusion seems to be in contradiction with the recent data of Clark et al..$^{8}$ who found no relationship between the development of cloudiness and gel formation, although turbidity effects were ascribed to helix-helix aggregation which was described as a condition for network formation and gelation'. Such a discrepancy may arise from a difference in the methods employed to prepare gels. Turbidity depends on the size of aggregates which may vary with the molecular weight and the preparation procedure.

The variation of $G^{\prime}$ as a function of concentration is often discussed for physical gels obtained from dilute solutions of synthetic or biological polymers. The results obtained with amylose in $0.2 \mathrm{M} \mathrm{KCl}$ are consistent with those reported by Ellis and Ring ${ }^{6}$ although the samples and methods of preparation of the gels were different. The marked concentration dependence can be ascribed simply to the fact that the range of concentrations investigated is in the vicinity of the limiting 
concentration $\left(C_{0}\right)$ for gelation to occur. True gels were obtained for concentrations as low as $1 \%$, so that $C_{0}$ was $\sim 0.9 \%$ in $\mathrm{KCl}$ medium regardless of the ionic strength. Miles et al. ${ }^{5}$ suggested that $C_{0}$ should have the same order of magnitude as the overlap concentration $\left(C^{*}\right)$. This last parameter was estimated from viscosity measurements to correspond to $C^{*}[\eta]=1.5^{5}$ or $1.8^{9}$, and thus varies with the molecular weight. The amylose sample studied here had an intrinsic viscosity in $0.33 \mathrm{M} \mathrm{KCl}$ of $0.7 \mathrm{dV} / \mathrm{g}$, which yields $C^{*} 2-2.5 \%$ depending on the criterion used. This value is thus much higher than that $(0.9 \%)$ of $C_{0}$. The present data thus contradict the suggestion by Miles et al. ${ }^{5}$, but are consistent with values reported recently, $C_{0}$ being 1.0 $\pm 0.2 \%$ regardless of the molecular weight ${ }^{8,9}$. This view is in line with arguments ${ }^{22}$ based on a theoretical treatment for describing the variation of $G^{\prime}$ with concentration. These authors predicted that $C_{0}$ is not equivalent to $C^{*}$ and can be much smaller than would be expected from overlap volumes of macromolecular coils. The discrepancy between our $C_{0}$ value and that reported by Miles et al. ${ }^{5}$ is explained easily when considering Fig. 8. The lowest concentration they investigated was $1.5 \%$ with a rigidity modulus of $\sim 50 \mathrm{~Pa}$, which is far removed from the actual $G^{\prime}$ values found here for the less concentrated gels $\left(1 \%, G^{\prime} \sim 5 \mathrm{~Pa}\right)$. The fact that $C_{0}$ seems to be independent of the molecular weight of the amylose, as suggested by the recently reported data ${ }^{8,9}$ and the present results, is not consistent with what is expected for synthetic polymers for which significant chain overlap is a necessary condition for gelation ${ }^{27}$. This is made possible when cross-links have finite energy ${ }^{22}$, and this condition is probably fulfilled ${ }^{9}$ for amylose gels which melt in the temperature range $140-160^{\circ 31}$.

The marked effect of ionic strength on the kinetics of gelation and on the final gel characteristics was not expected since intrinsic viscosity in neutral solution is not affected by the ionic strength, at least up to $\mathrm{M}$. However, Kitamura et al. ${ }^{13}$ observed an increase in the final size of particles when the content of $\mathrm{KCl}$ was increased and suggested that the number of nuclei would decrease with increase in the concentration of salt, thus slowing the rate of retrogradation. The present data are consistent with such a suggestion, assuming that the number of interconnecting points depends on the content of $\mathrm{KCl}$. Furthermore, since the number of cross-links is reduced, a gel obtained at a given concentration is weaker when the content of $\mathrm{KCl}$ increases due to a reduced amount of interconnections.

\section{ACKNOWLEDGMENTS}

We thank the NATO Scientific Grant Program for the research grant (to JLD) which enabled this work to be carried out at Quebec, the Université Laval for partial support, and M.A.V. Axelos (INRA-Nantes) for helpful discussions.

\section{REFERENCES}

1 M. J. Milles, V. J. Morris, P. D. ORFord, AND S. G. RING, Carbohydr. Res., 135 (1985) 271-281.

2 S. G. Ring, Staerke, 37 (1985) 80-83. 
3 J. R. Katz, Z. Phys. Chem., 150 (1930) 37-59. 4 R. Collison, in J. A. RADLEY (Ed.), Starch and its Derivatives, Chapman and Hall, London, 1968,

5 M. J. Miles, V. J. Morris, ANd S. G. Ring, Carbohydr. Res., 135 (1985) 257-269.

6 H. S. Eluis AND S. G. Ring, Carbohydr. Polym., 5 (1985) 201-213.

7 M. J. GiDLEY AND P. V. Bulpin, Macromolecules, 22 (1989) 341-346.

8 A. H. Clark, M. J. Gidley, R. K. Richardson, and S. B. Ross-Murphy, Macromolecules, 22 (1989) 346-351.

9 M. J. GIDLEY, Macromolecules, 22 (1989) 351-358.

10 E. F. Paschall and J. F. Foster, J. Polym. Sci., 9 (1953) 73-84.

11 J. F. Foster and M. D. Sterman, J. Polym. Sci., 21 (1956) 91-101.

12 E. Huseman, B. Pfannemuller, and W. Burchard, Makromol. Chem., 59 (1963) 1-27.

13 S. Kitamura, S. Yoneda, And T. Kuge, Carbohydr. Polym., 4 (1984) 127-136.

14 A. Hayash, K. Kinoshita, AND Y. Mryake, Polym. J., 13 (1981) 537-541.

15 A. Hayashi, K. Kinoshita, Y. Mryake. and C. H. Cho, Agric. Biol. Chem., 47 (1983) 1699-1704.

16 S. G. RING, K. J. I'ANSON, AND V. J. MoRRIs, Macromolecules, 18 (1985) 182-188.

17 W. W. Everett AND J. F. Foster, J. Am. Chem. Soc., 81 (1959) 3464-3469.

18 W. Banks and C. T. Greenwood, Makromol. Chem., 67 (1963) 49-63.

19 H. H. Winter AND F. Chambon, J. Rheol., 30 (1986) 367-382.

20 H. H. Winter, Polym. Eng. Sci., 27 (1987) 1698-1702.

21 M. Djabourov, J. Leblond, and P. Papon, J. Phys. (Paris), 49 (1988) 333-343.

22 A. H. Clark and S. B. Ross-Murphy, Eur. Polym. J., 17 (1985) 164-168.

23 R. K. Richardson, G. Roginson, S. B. Ross-Murphy, and S. Todo, Polym. Bull 4 (1981) 541 546.

24 W. Banks and C. T. Greenwood, Starch and its Components, University Press, Edinburgh, 1975. 25 V. S. RAO AND J. F. Foster, Biopolymers, 1 (1963) 527-544.

26 H. L. Doppert and A. J. Staverman, J. Polym. Sci., Part Al, 4 (1966) 2353-2366.

27 M. J. MrLes, in D. C. BAssetT (Ed.), Development in Crystalline Polymers-2, Elsevier Applied Science, New York, 1988, pp. 233-295.

28 S. Wellinghoff, J. SHAW, AND E. BAER, Macromolecules, 12 (1979) 932-939.

29 J. M. GUENET, B. Lotz, AND J. C. WitTMANn, Macromolecules, 18 (1985) $420-427$.

30 K. J. I'Anson, M. J. Mines, V. J. Morris, AND S. G. Ring, Carbohydr. Polym., 8 (1988) 45-53.

31 A. H. Clark and S. B. Ross-Murphy, Adv. Polym. Sci., 83 (1987) 57-192.

32 K. Eberstein, R. Hopke, G. KonieCZNY-JANDA, AND R. STUTE, Staerke, 32 (1980) 397-404. 